Artículo científico

Volumen 33(2): Artículo 46938, 2022

e-ISSN 2215-3608, doi:10.15517/am.v33i2.46938

https://revistas.ucr.ac.cr/index.php/agromeso/index

\title{
Subpoblaciones morfométricas de espermatozoides epididimarios del venado de cola blanca (Odocoileus virginianus peruvianus) ${ }^{1}$
}

\section{Morphometric subpopulations study of white-tailed deer (Odocoileus virginianus peruvianus) epididymal spermatozoa}

\author{
Hernán Cucho², Gregorio Nina², Aydee Meza', Ruth Ccalta², César Ordóñez ${ }^{2}$, Anthony Valverde ${ }^{3}$
}

1 Recepción: 14 de mayo, 2021. Aceptación: 9 de septiembre, 2021. Este trabajo formó parte de la tesis de Licenciatura en Zootecnia del segundo autor, desarrollada en el Laboratorio de Biotecnologías Reproductivas de la Escuela Profesional de Zootecnia en el Centro Experimental La Raya - Cusco, de la Universidad Nacional de San Antonio Abad del Cusco, Cusco, Perú.

2 Universidad Nacional de San Antonio Abad del Cusco, Facultad de Ciencias Agrarias, Escuela Profesional de Zootecnia, Laboratorio de Biotecnologías Reproductivas, Granja K’ayra S/N, San Jerónimo, Centro Experimental La Raya - Cusco. Apdo. Postal 921, Cusco, Perú. hernan.cucho@unsaac.edu.pe (https://orcid.org/0000-0001-7170-9795); gregorioninanina@gmail.com (https://orcid.org/0000-0003-14541417); aydee.meza@unsaac.edu.pe (https://orcid.org/0000-0001-8581-9987); ruthccaltahancco@ gmail.com (https://orcid.org/0000-00023730-4468); cesar.ordoñez@unsaac.edu.pe (https://orcid.org/0000-0002-2955-4555).

3 Instituto Tecnológico de Costa Rica (ITCR), Escuela de Agronomía, Centro de Investigación y Desarrollo en Agricultura Sostenible del Trópico Húmedo, Laboratorio de Reproducción Animal, Campus Tecnológico Local San Carlos. Apdo. Postal 223-21002 Alajuela, Costa Rica. anvalverde@tec.ac.cr (autor para correspondencia; https://orcid.org/0000-0002-3191-6965).

\section{Resumen}

Introducción. El estudio de la morfometría espermática del venado cola blanca (Odocoileus virginianus peruvianus) permite caracterizar las células espermáticas de esta especie con propósitos de conservación. Objetivo. Determinar las subpoblaciones espermáticas según la morfometría de los espermatozoides epididimarios del venado de cola blanca, con un sistema CASA-Morph. Materiales y métodos. El estudio se desarrolló en el segundo semestre 2016, en la Universidad Nacional de San Antonio Abad del Cusco, Perú. En muestras de semen obtenidas del extremo caudal del epidídimo de dos venados (dos y cuatro dientes), se determinó con base en el sistema CASAMorph, Integrated Semen Analysis System (ISAS ${ }^{\circledR} \mathrm{v} 1$ ): la longitud, anchura, área, perímetro, elipticidad, elongación, regularidad y rugosidad de la cabeza del espermatozoide, también, la anchura, área, distancia y ángulo de inserción de la pieza intermedia. Se realizó un análisis de componentes principales (PCA) y se aplicó el modelo no jerárquico k-medias para determinar el número óptimo de clústeres. Resultados. Las variables morfométricas se distribuyeron en cinco PCA: elipticidad, tamaño, circularidad, ángulo de inserción de la pieza intermedia y anchura de la pieza intermedia, que explicaron un 81,24\% de la varianza total. El análisis de clústeres determinó cuatro subpoblaciones (SP), la SP1 agrupó células grandes, alargadas con alta elongación y elipticidad $(21,76 \%)$, la SP2 compuesta por espermatozoides de tamaño pequeño y delgados tanto en la cabeza como en la pieza intermedia (37,7 \%), la SP3 se relacionó con la presencia de células alargadas con valores altos de anchura de la pieza intermedia, elipticidad y elongación (17,7\%), y la SP4 agrupó espermatozoides de tamaño intermedio de la cabeza y de la pieza intermedia $(22,84 \%)$. Conclusiones. La determinación de cuatro subpoblaciones espermáticas en el semen de venado cola blanca puede ser la base de programas de reproducción asistida de esta especie.

Palabras clave: semen de venado, CASA morph, reproducción animal, recursos genéticos silvestres de animales. 


\begin{abstract}
Introduction. The study of the sperm morphometry of the white-tailed deer (Odocoileus virginianus peruvianus) allows to characterize the sperm cells of this species for conservation purposes. Objective. To determine the sperm subpopulations according to the morphometry of the epididymal spermatozoa of the white-tailed deer, using a CASA-Morph system. Materials and methods. The study was carried out in the second semester 2016, at the Universidad Nacional San Antonio Abad del Cusco, Peru. In semen samples obtained from the caudal end of the epididymis of two deer (two and four teeth), it was determined based on the CASA-Morph system, Integrated Semen Analysis System (ISAS ${ }^{\circledR} \mathrm{v} 1$ ): the length, width, area, perimeter, ellipticity, elongation, regularity, and roughness of the sperm head were determined, also, the width, area, distance, and midpiece insertion angle of the sperm. Principal components analysis (PCA) was performed and the non-hierarchical k-means model was applied to determine the optimal number of clusters. Results. The morphometric variables were distributed in five PCAs: ellipticity, size, circularity, midpiece insertion angle, which explained $81.24 \%$ of the total variance. The Cluster analysis determined four subpopulations (SP), SP1 grouped large, elongated cells with high elongation and ellipticity (21.76\%), SP2 composed of small and thin spermatozoa both in the head and in the midpiece $(37.7 \%)$. The SP3 was related to the presence of elongated cells with high values of midpiece width, ellipticity, and elongation (17.7\%), and SP4 grouped spermatozoa with intermediate size of the head and the midpiece $(22.84 \%)$. Conclusion. The determination of four sperm subpopulations in white-tailed deer semen can be the basis of assisted reproduction programs for this species.
\end{abstract}

Keywords: deer semen, CASA morph, animal reproduction, animal reproduction, wild animal genetic resources.

\title{
Introducción
}

Los venados de cola blanca, son uno de los grupos de mamíferos más numerosos en América, su población se estima entre ocho y quince millones de individuos. En el continente se conocen 38 subespecies, 30 en el norte y Centroamérica y ocho en Sudamérica (Ramírez Lozano, 2012). En el Perú la única subespecie reportada es la Odocoileus virginianus peruvianus (Pacheco et al., 2009); en este país, el venado de cola blanca no figura en el listado de especies en peligro de extinción (Ministerio de Desarrollo Agrario y Riego del Perú, 2014); sin embargo, al ser una especie silvestre protegida por el estado, es necesario obtener permisos para su estudio, que en el área reproductiva son escasos, por la dificultad de obtener muestras.

Las referencias sobre las características seminales del O. virginianus peruvianus, son escasas y se limitan a descripciones generales; las de morfometría de los espermatozoides son inexistentes. En Colombia se reportan trabajos en donde han evaluado las características de movilidad y morfometría espermática en la subespecie colombiana del venado de cola blanca (Hernández-Corredor et al., 2018), también hay reportes de movilidad en subespecies de Estados Unidos (Ake-Lopez et al., 2010; Kirschner \& Rodenkirch, 2017).

A pesar de que los espermatozoides adquieren su capacidad fertilizante y sus propiedades de movilidad durante el tránsito por el epidídimo (Gaddum, 1968; Orgebin-Crist, 1967), se ha descrito que los espermatozoides recogidos de la cola del epidídimo son maduros a nivel funcional, tienen la capacidad de fertilización similar a los espermatozoides provenientes de un eyaculado (Robaire et al., 2015). Durante el proceso de tránsito epididimario, las modificaciones a las que es expuesto el gameto masculino se denominan maduración espermática (Cooper \& Yeung, 2006). Además, el epidídimo está relacionado con la concentración de espermatozoides en el eyaculado, procesos de inmuno protección celular y una función de reserva espermática (Belleannée et al., 2012; Cornwall, 2009). 
El desarrollo de los sistemas CASA-Morph (Computer Assisted Semen Analysis) para el estudio de la morfometría de la cabeza y pieza intermedia de los espermatozoides, abre nuevas posibilidades para la evaluación seminal (Soler et al., 2016; Valverde, Castro-Morales, 2019; Yániz, Soler et al., 2015). El examen de la morfometría espermática con los sistemas de análisis computarizado de semen (CASA - Morph), aporta información precisa y objetiva de las variables morfométricas de los espermatozoides de una muestra de semen (Martí et al., 2012; Valverde et al., 2020). Diversos estudios sobre morfometría espermática han indicado variaciones importantes en animales de una misma población (Soler, Alambiaga et al., 2017; Thurston et al., 2001) e incluso entre eyaculados de un mismo animal (Valverde et al., 2016). En otras especies, los trabajos realizados por Cucho et al. (2019) en alpaca (Vicugna pacos), Cucho et al. (2021) en llama (Lama glama), Soler et al. (2005) en ciervo rojo ibérico (Cervus elaphus hispanicus), Tuset et al. (2008) en trucha arcoíris (Oncorhynchus mykiss) y Esteso et al. (2015) en Ibex ibérico (Capra pyrenaica) han descrito que el tipo de tinción tiene influencia sobre las medidas morfométricas del espermatozoide.

Se han descrito relaciones entre la morfometría espermática y la fertilidad de los machos (Downing-Meisner et al., 2005; Gage, 1998; Maroto-Morales et al., 2016; Peña et al., 2005), las cuales señalan que al evaluar el tamaño y forma de la cabeza del espermatozoide, se podrían detectar alteraciones en la maduración epididimaria durante la espermatogénesis (Hidalgo et al., 2008).

La gran cantidad de células analizadas con los sistemas CASA permiten disponer de información de los espermatozoides individuales y utilizar esos grandes volúmenes de datos para realizar procedimientos multivariados de análisis para determinar diferentes patrones morfométricos en el semen (Barquero, Roldan et al., 2021; Yániz et al., 2016). En los últimos años, la información proporcionada por los sistemas CASA, combinada con la estadística multivariada, ha mostrado la verdadera estructura de la población espermática en diferentes especies, la cual está compuesta por diferentes subpoblaciones (Barquero, Víquez et al., 2021; Soler et al., 2016; Soler, Contell et al., 2017; Valverde et al., 2018; Valverde, Castro-Morales 2019; Valverde, Madrigal-Valverde et al., 2019; Valverde et al., 2021; Víquez et al., 2020; Yániz, Palacín et al., 2015). La existencia de estas subpoblaciones confirma la idea que los eyaculados de mamíferos, peces y reptiles son heterogéneos y que esta distribución de subpoblaciones de espermatozoides pueden tener diferentes respuestas a estímulos funcionales (Martinez-Pastor, Diaz-Carujo et al., 2005), patrones de movilidad (Martinez-Pastor, Garcia-Macias et al., 2005; Ramón et al., 2012; 2013) y diferentes descriptores morfométricos (Beracochea et al., 2014; Esteso et al., 2018; Ramón et al., 2013). Existen estudios sobre morfometría y subpoblaciones espermáticas en especies similares al venado de cola blanca, como es el venado de las pampas (Beracochea et al., 2014), y el ciervo rojo ibérico (Esteso et al., 2009; Ramón et al., 2013).

El propósito de este trabajo fue determinar las subpoblaciones espermáticas según la morfometría de los espermatozoides epididimarios del venado cola blanca, con un sistema CASA-Morph.

\section{Materiales y métodos}

\section{Descripción del experimento}

El venado de cola blanca (Odocoileus virginianus peruvianus), es una especie protegida por el Estado Peruano; por ello, el Servicio Nacional Forestal y de Fauna Silvestre, por Resolución N 235-2015-SERFOR/DGGSPFFS, autorizó el uso de muestras de testículos de ejemplares de la especie en estudio, provenientes de decomisos y caza deportiva. 


\section{Localización del estudio y animales}

El estudio se realizó en el Centro Experimental La Raya, Granja K'ayra y Laboratorio de Biotecnologías Reproductivas de la Escuela Profesional de Zootecnia, Faculta de Ciencias Agrarias, de la Universidad Nacional de San Antonio Abad del Cusco (UNSAAC), durante los meses de junio a diciembre del año 2016 y las muestras provenientes de animales de caza deportiva se colectaron durante los dos primeros meses. Se utilizaron dos venados de entre tres y cuatro años de edad, peso de 54,15 \pm 16,05 kg. Ambos ejemplares procedían de las provincias de Paruro y Quispicanchi, Cusco, Perú. Se extirparon los testículos, epidídimos con sus envolturas escrotales, material que se transportó en un frigorífico portátil a $15^{\circ} \mathrm{C}$, desde el lugar de captura hasta el Laboratorio de Biotecnologías Reproductivas, en el Centro Experimental La Raya, a 4130 m.s.n.m. El tiempo de traslado medio fue de 4 h.

\section{Recuperación de espermatozoides}

En el laboratorio Laboratorio de Biotecnologías Reproductivas de la UNSAAC, se realizó la limpieza de las envolturas testiculares que recubren el testículo y epidídimo, según lo descrito por Peña Hernández (2014), luego se realizó el pesado $(22,4$ y $23,7 \mathrm{~g})$ y medición de las dimensiones testiculares $(6,5 \pm 0,4$ x 2,7 $\pm 0,3 \mathrm{~cm})$. La recuperación de espermatozoides se realizó de la cola del epidídimo (de una longitud de 8,9 y 10,3 cm), con incisiones sobre esta (Martinez-Pastor et al., 2006; Soler et al., 2005) con una hoja de bisturí, se arrastró el material espermático a una placa Petri, donde se había depositado $1 \mathrm{~mL}$ de diluyente base Tris (Tris $0,30 \mathrm{~g}$, ácido cítrico $0,17 \mathrm{~g}$, fructuosa $0,13 \mathrm{~g}$, agua destilada hasta completar $10 \mathrm{~mL}$ ) (Souza, 2009).

\section{Evaluación de espermatozoides}

Los espermatozoides mezclados con el diluyente, se vertieron en un tubo de $2 \mathrm{~mL}$, que se mantuvo a $37^{\circ} \mathrm{C}$ en un baño seco. Una muestra de $5 \mu \mathrm{L}$ se colocó en un portaobjetos para confirmar la presencia de espermatozoides. Para el estudio de la morfometría del espermatozoide del venado de cola blanca, se realizaron ocho frotis por cada animal, con $5 \mu \mathrm{L}$ de muestra, que se tiñó con Hemacolor (Merck, Darmstadt, Alemania), según protocolo del producto. El análisis de morfometría espermática de las muestras se realizó con el sistema ISAS ${ }^{\circledR}$ v1 CASA-Morph (Proiser R+D, Paterna, España). Las células espermáticas se capturaron con un microscopio de contraste de fase (UOP; Proiser R+D) equipado con un objetivo de 100x de campo claro (AN 0.17) y una cámara digital de video (Proiser 782M). El tamaño de las cuadrículas de captura de video fue de 768x576x8 bits con 256 niveles de grises. La resolución de las imágenes fue de $0,084 \mu \mathrm{m} /$ pixel en ambos ejes. Se capturaron y analizaron 740 imágenes de espermatozoides de ambos animales. Se analizaron los siguientes parámetros morfométricos: longitud (L, $\mu \mathrm{m})$, anchura $(\mathrm{W}, \mu \mathrm{m})$, área $\left(\mathrm{A}, \mu \mathrm{m}^{2}\right)$ y perímetro $(\mathrm{P}, \mu \mathrm{m})$. Además, se evaluaron cuatro variables de forma de la cabeza del espermatozoide:

a) La elipticidad que se obtuvo mediante la fórmula (L/W) y describe la forma elíptica de un objeto, de forma que su valor es uno para un círculo y mayor que uno para una elipse. Cuando los valores son superiores a la unidad, la forma de la cabeza del espermatozoide tiende a ser más delgada.

b) La elongación que se obtuvo mediante la fórmula $([\mathrm{L}-\mathrm{W}] /[\mathrm{L}+\mathrm{W}])$ y describe el grado de alargamiento de un objeto, por lo que su valor es cero para un círculo y alcanza un valor de uno para una línea. Si este valor aumenta, la cabeza del espermatozoide tiende a ser más alargada. 
c) La regularidad ( $\pi \mathrm{LW} / 4 \mathrm{~A})$ que describe el grado de uniformidad de la forma de la cabeza del espermatozoide.

d) La rugosidad $\left(4 \pi \mathrm{A} / \mathrm{P}^{2}\right)$ que describe el grado de circularidad de un objeto. Cuando se tiene un círculo ideal el valor es uno, y cero cuando es una línea. Si el valor tiende a uno, la cabeza del espermatozoide presenta una forma más circular. También se midió la anchura $(\mu \mathrm{m})$, área $\left(\mu \mathrm{m}^{2}\right)$, distancia de inserción (distancia entre la cabeza y el eje de la pieza intermedia, $\mu \mathrm{m}$ ) y ángulo de inserción $\left(^{\circ}\right)$ de la pieza intermedia del espermatozoide.

\section{Análisis estadístico}

En las variables de morfometría, se determinaron los supuestos de normalidad y homocedasticidad mediante las pruebas de Shapiro-Wilks y Levene para los datos obtenidos del análisis CASA-Morph de todas las células analizadas. Se realizaron procedimientos de agrupamiento para identificar subpoblaciones espermáticas del conjunto de datos de morfometría. Todos los valores para las variables morfométricas se estandarizaron para evitar cualquier efecto de escala. El primer proceso consistió en realizar un análisis de componentes principales (PCA) o análisis factorial de todos los datos para obtener un menor número de combinaciones lineales (PC) que aún conservaran la mayor cantidad de información posible de las variables originales.

El número de componentes principales (PCA) utilizados en el proceso del análisis se determinó a partir del criterio de Kaiser. Se seleccionaron solo aquellos con un valor propio (eingenvalue; varianza extraída de cada PC $>1$ ). Además, se realizó la prueba de esfericidad de Bartlett y la prueba KMO (Kaiser-Meyer-Olkin) (Spencer, 2013). Como método de rotación, se utilizó el método varimax con la normalización de Kaiser (Kaiser, 1958).

El segundo proceso consistió en realizar un análisis no jerárquico con el modelo de k-medias (k-means) que utiliza distancias euclidianas de las variables cuantitativas después de la estandarización de los datos, por lo que los centros de agrupación fueron las medias de las observaciones asignadas a cada agrupación (Kaufman \& Rousseeuw, 2005). El análisis multivariado de grupos de k-medias se realizó para clasificar los espermatozoides en un número reducido de subpoblaciones (clusters o grupos) de acuerdo con sus variables morfométricas. En el proceso final, para determinar el número óptimo de grupos, los centroides finales se agruparon a nivel jerárquico con el método Ward (Murtagh \& Legendre, 2014). Por lo tanto, el procedimiento de agrupamiento permite la identificación de subpoblaciones de espermatozoides, ya que cada grupo contribuyó a un grupo final formado por los espermatozoides unidos a los centroides. Se realizó un análisis de la varianza (ANOVA) para evaluar las diferencias estadísticas en las distribuciones de observaciones dentro de las subpoblaciones de espermatozoides para cada variable morfométrica. Las diferencias entre medias se analizaron mediante la prueba de Bonferroni. Los resultados se presentan como media \pm error estándar de la media (SEM). La significación estadística se consideró en $\mathrm{P}<0,05$. Todos los datos se analizaron con el paquete IBM SPSS, versión 23.0 para Windows (SPSS Inc., Chicago, IL, EE. UU.).

\section{Resultados}

El rango de la longitud de testículos de los animales estudiados fue entre 6,2 y $6,8 \mathrm{~cm}$, mientras que la anchura presentada por los animales fue de $2,5 \mathrm{~cm}$ y $2,9 \mathrm{~cm}$. La longitud del epidídimo en los machos estudiados se determinó en 8,9 y $10,3 \mathrm{~cm}$. Se presentó un efecto animal $(\mathrm{p}<0,05)$ en las variables morfométricas de cabeza y pieza intermedia de los espermatozoides. Las variables morfométricas que indicaron la mayor variabilidad correspondieron a la pieza intermedia y fueron la anchura, área, distancia y ángulo de inserción, con coeficientes de variación de 44,65 \%,22,99\%, 76,81\% y $81,83 \%$, respectivamente. Se presentaron diferencias $(\mathrm{p}<0,05)$ entre 
animales para las variables de morfometría, las variables de la pieza intermedia del espermatozoide fueron las que mostraron mayores diferencias entre los animales (Cuadro 1).

Cuadro 1. Variables morfométricas de la cabeza y pieza intermedia (media \pm DE) del espermatozoide de venado de cola blanca (Odocoileus virginianus peruvianus). Laboratorio de Biotecnologías Reproductivas, Granja K'ayra, Escuela Profesional de Zootecnia, Facultad de Ciencias Agrarias de la Universidad Nacional de San Antonio Abad del Cusco (UNSAAC), Cusco, Perú, 2016.

Table 1. Morphometric variables of the head and intermediate piece (mean $\pm \mathrm{SD}$ ) of the white-tailed deer (Odocoileus virginianus peruvianus) sperm. Reproductive Biotechnologies Laboratory, K'ayra Farm, Professional School of Animal Sciences, Faculty of Agrarian Sciences at Universidad Nacional de San Antonio Abad in Cuzco (UNSAAC), Cusco, Peru, 2016.

\begin{tabular}{lccc}
\hline & & \multicolumn{2}{c}{ Venado } \\
\cline { 3 - 4 } Variable & Promedio & $\mathbf{1}$ & $\mathbf{2}$ \\
\hline Longitud cabeza $(\mu \mathrm{m})$ & $7,63 \pm 0,45$ & $7,62 \pm 0,47$ & $7,64 \pm 0,45$ \\
Anchura cabeza $(\mu \mathrm{m})$ & $3,87 \pm 0,18$ & $3,80 \pm 0,14$ & $3,88^{*} \pm 0,18$ \\
Área cabeza $\left(\mu \mathrm{m}^{2}\right)$ & $25,43 \pm 1,89$ & $25,13 \pm 1,60$ & $25,48 \pm 1,94$ \\
Perímetro cabeza $(\mu \mathrm{m})$ & $22,19 \pm 1,49$ & $22,01 \pm 1,24$ & $22,23 \pm 1,53$ \\
Elipticidad & $1,98 \pm 0,12$ & $2,01 * \pm 0,14$ & $1,97 \pm 0,12$ \\
Rugosidad & $0,65 \pm 0,07$ & $0,66 \pm 0,06$ & $0,65 \pm 0,07$ \\
Elongación & $0,33 \pm 0,03$ & $0,334^{*} \pm 0,03$ & $0,326 \pm 0,03$ \\
Regularidad & $0,91 \pm 0,03$ & $0,90 \pm 0,03$ & $0,91^{*} \pm 0,03$ \\
Anchura PI $(\mu \mathrm{m})$ & $1,50 \pm 0,67$ & $1,34 \pm 0,70$ & $1,53^{*} \pm 0,66$ \\
Área PI $\left(\mu \mathrm{m}^{2}\right)$ & $3,57 \pm 0,82$ & $3,62 \pm 0,73$ & $3,56 \pm 0,83$ \\
Distancia inserción PI $(\mu \mathrm{m})$ & $0,18 \pm 0,14$ & $0,15 \pm 0,13$ & $0,18^{*} \pm 0,14$ \\
Ángulo inserción PI $\left({ }^{\circ}\right)$ & $3,88 \pm 3,17$ & $3,24 \pm 2,55$ & $3,99^{*} \pm 3,26$ \\
\hline
\end{tabular}

Número total de espermatozoides de cada animal: venado $1=108$; venado $2=632$. PI: pieza intermedia. DE: desviación estándar. * indica diferencias significativas entre animales $\mathrm{p}<0,05$. / Total number of sperm cells from each animal: deer $1=108$; deer $2=632$. PI: mid piece. SD: standard deviation. * indicates significant differences between animals $\mathrm{p}<0.05$.

Los resultados del análisis de componentes principales indicaron que había cinco componentes principales (PCs): "elipticidad" (PC1) representada por la elongación, elipticidad, la anchura y longitud de la cabeza del espermatozoide. El efecto del valor propio de las dos primeras variables fue el mismo $(0,989)$, mientras que para la anchura de la cabeza fue de $-0,488$, lo que indica que en este grupo se incluyeron aquellas células con una cabeza alargada pero delgada. PC2 se refería a anchura, longitud, área y regularidad de la cabeza, llamado "tamaño" con un mayor efecto por área de la cabeza. En este grupo hubo células con una longitud de cabeza similar al grupo anterior, pero más anchas por efecto del área total de la cabeza respecto del grupo celular comprendido en PC1. PC3 estuvo representado por el perímetro y la rugosidad, denominada "circularidad" dada por rugosidad $(-0,966)$. El PC4 estuvo representado por el ángulo de inserción $(0,796)$ y distancia de la pieza intermedia, denominado "ángulo de inserción de la pieza intermedia”. El PC5 lo comprendió la anchura y el área de la pieza intermedia, llamado “anchura de la pieza intermedia". Los 5 PC tuvieron una variación total explicada de 81,24 \%. Estos resultados 
indicaron que la forma y el tamaño del espermatozoide tuvo un efecto mayor sobre la varianza total que las otras variables (Cuadro 2).

Cuadro 2. Valores propios de componentes principales (CPs) para variables morfométricas en espermatozoides de venado de cola blanca (Odocoileus virginianus peruvianus). Laboratorio de Biotecnologías Reproductivas, Granja K'ayra, Escuela Profesional de Zootecnia, Facultad de Ciencias Agrarias de la Universidad Nacional de San Antonio Abad del Cusco (UNSAAC), Cusco, Perú, 2016.

Table 2. Eigen values of principal components (PCs) for morphometric variables in white-tailed deer (Odocoileus virginianus peruvianus) sperm. Reproductive Biotechnologies Laboratory, K'ayra Farm, Professional School of Animal Sciences, Faculty of Agrarian Sciences at Universidad Nacional de San Antonio Abad in Cuzco (UNSAAC), Cusco, Peru, 2016.

\begin{tabular}{|c|c|c|c|c|c|}
\hline \multirow[b]{2}{*}{ Variable } & \multicolumn{5}{|c|}{ Componentes principales } \\
\hline & PC1 & PC2 & PC3 & PC4 & PC5 \\
\hline Elongación & 0,989 & & & & \\
\hline Elipticidad & 0,989 & & & & \\
\hline Anchura cabeza & $-0,488$ & 0,864 & & & \\
\hline Longitud cabeza & 0,694 & 0,693 & & & \\
\hline Área cabeza & & 0,878 & & & \\
\hline Regularidad & & 0,420 & & & \\
\hline Rugosidad & & & $-0,966$ & & \\
\hline Perímetro cabeza & & & 0,898 & & \\
\hline Anchura PI & & & & & 0,757 \\
\hline Área PI & & & & & 0,752 \\
\hline Distancia inserción PI & & & & 0,756 & \\
\hline Ángulo inserción PI & & & & 0,796 & \\
\hline Varianza explicada (\%) & 28,34 & 19,75 & 13,59 & 10,33 & 9,23 \\
\hline
\end{tabular}

Varianza total explicada $=81,24 \%$. PI: pieza intermedia. / Total variance explained $=81.24 \%$. PI: mid piece.

Los datos del análisis de conglomerados mostraron cuatro subpoblaciones (SP) (Figura 1 y Cuadro 3). Los valores morfométricos correspondientes a cada subpoblación se caracterizaron por: (a) la cabeza de los espermatozoides incluidos en esta subpoblación presentaron la mayor longitud, área y perímetro, y fueron las más elípticas, elongadas y regulares. Su pieza intermedia resultó la de menor anchura $(1,01 \pm 0,51)$ y contuvo el $21,76 \%$ del total de células (Figura 2a y Cuadro 3). (b) Espermatozoides con un tamaño de cabeza más pequeño y delgado (SP2), tanto en la cabeza como en la pieza intermedia, exhibió longitudes de cabeza y anchura bajas (7,39 $\pm 0,28 \mu \mathrm{m} ; 3,77 \pm 0,13 \mu \mathrm{m}$ ), y estuvo constituido por el 37,7 \% de los espermatozoides (Figura $2 \mathrm{~b}$ y Cuadro 3). (c) Espermatozoides con cabezas alargadas y con valores altos de anchura de la pieza intermedia (SP3; 7,63 $\pm 0,28 \mu \mathrm{m} ; 1,81 \pm 0,66 \mu \mathrm{m})$. Además, esta subpoblación presentó células con valores intermedios de elipticidad y elongación, representó el 17,7 \% del total de células (Figura 2c y Cuadro 3), y (d) células de tamaño intermedio de la cabeza $\left(26,11 \pm 1,80 \mu \mathrm{m}^{2}\right)$ y pieza intermedia (SP4), con valores bajos de elipticidad y elongación $(1,84 \pm 0,08$; $0,30 \pm 0,02$ ), representó el 22,84\% del total de células (Figura 2d y Cuadro 3). Se observó que las SP1, SP2 y SP3 mostraron proporciones similares $(17,70$ a $22,84 \%)$. 


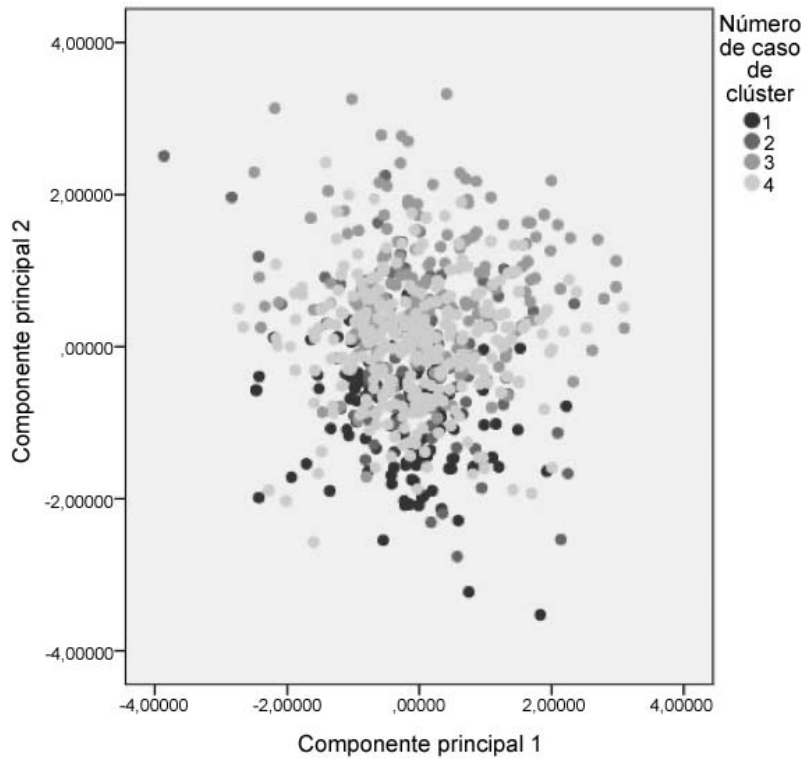

Figura 1. Distribución de espermatozoides en venado de cola blanca (Odocoileus virginianus peruvianus) en subpoblaciones según los valores de sus componentes principales (PCs). Laboratorio de Biotecnologías Reproductivas, Granja K'ayra, Escuela Profesional de Zootecnia, Facultad de Ciencias Agrarias de la Universidad Nacional de San Antonio Abad del Cusco (UNSAAC), Cusco, Perú, 2016.

Figure 1. Distribution of white-tailed deer (Odocoileus virginianus peruvianus) spermatozoa in subpopulations according to the values of principal components (PCs). Reproductive Biotechnologies Laboratory, K'ayra Farm, Professional School of Animal Sciences, Faculty of Agrarian Sciences at Universidad Nacional de San Antonio Abad in Cuzco (UNSAAC), Cusco, Peru, 2016.

Cuadro 3. Subpoblaciones espermáticas para variables morfométricas (media \pm DE) en venado de cola blanca (Odocoileus virginianus peruvianus). Laboratorio de Biotecnologías Reproductivas, Granja K'ayra, Escuela Profesional de Zootecnia, Facultad de Ciencias Agrarias de la Universidad Nacional de San Antonio Abad del Cusco (UNSAAC), Cusco, Perú, 2016.

Table 3. Sperm subpopulations for morphometric variables (mean $\pm \mathrm{SD}$ ) in white-tailed deer (Odocoileus virginianus peruvianus). Reproductive Biotechnologies Laboratory, K'ayra Farm, Professional School of Animal Sciences, Faculty of Agrarian Sciences at Universidad Nacional de San Antonio Abad in Cuzco (UNSAAC), Cusco, Perú, 2016.

\begin{tabular}{lcccc}
\hline & \multicolumn{4}{c}{ Subpoblación espermática } \\
\cline { 2 - 4 } Número de células $(\%)$ & SP1 & SP2 & SP3 & SP4 \\
\cline { 2 - 4 } & $161(21,76)$ & $279(37,70)$ & $131(17,70)$ & $169(22,84)$ \\
\hline Longitud cabeza $(\mu \mathrm{m})$ & $8,24 \pm 0,28^{\mathrm{a}}$ & $7,39 \pm 0,28^{\mathrm{c}}$ & $7,63 \pm 0,28^{\mathrm{b}}$ & $7,46 \pm 0,38^{\mathrm{c}}$ \\
Anchura cabeza $(\mu \mathrm{m})$ & $3,89 \pm 0,15^{\mathrm{b}}$ & $3,77 \pm 0,13^{\mathrm{c}}$ & $3,81 \pm 0,16^{\mathrm{c}}$ & $4,04 \pm 0,15^{\mathrm{a}}$ \\
Área cabeza $\left(\mu \mathrm{m}^{2}\right)$ & $26,98 \pm 1,38^{\mathrm{a}}$ & $24,24 \pm 1,51^{\mathrm{d}}$ & $25,17 \pm 1,53^{\mathrm{c}}$ & $26,11 \pm 1,80^{\mathrm{b}}$ \\
Perímetro cabeza $(\mu \mathrm{m})$ & $22,94 \pm 1,10^{\mathrm{a}}$ & $21,12 \pm 0,89^{\mathrm{b}}$ & $22,93 \pm 1,75^{\mathrm{a}}$ & $22,70 \pm 1,34^{\mathrm{a}}$ \\
Elipticidad & $2,12 \pm 0,09^{\mathrm{a}}$ & $1,96 \pm 0,07^{\mathrm{c}}$ & $2,01 \pm 0,09^{\mathrm{b}}$ & $1,84 \pm 0,08^{\mathrm{d}}$ \\
Rugosidad & $0,65 \pm 0,05^{\mathrm{b}}$ & $0,68 \pm 0,05^{\mathrm{a}}$ & $0,61 \pm 0,09^{\mathrm{c}}$ & $0,64 \pm 0,07^{\mathrm{b}}$ \\
Elongación & $0,36 \pm 0,02^{\mathrm{a}}$ & $0,32 \pm 0,02^{\mathrm{c}}$ & $0,33 \pm 0,02^{\mathrm{b}}$ & $0,30 \pm 0,02^{\mathrm{d}}$ \\
Regularidad & $0,93 \pm 0,03^{\mathrm{a}}$ & $0,91 \pm 0,03^{\mathrm{b}}$ & $0,91 \pm 0,03^{\mathrm{b}}$ & $0,91 \pm 0,03^{\mathrm{b}}$ \\
Anchura PI $(\mu \mathrm{m})$ & $1,01 \pm 0,51^{\mathrm{c}}$ & $1,45 \pm 0,59^{\mathrm{b}}$ & $1,81 \pm 0,66^{\mathrm{a}}$ & $1,80 \pm 0,64^{\mathrm{a}}$ \\
Área PI $\left(\mu \mathrm{m}^{2}\right)$ & $3,74 \pm 0,68^{\mathrm{a}}$ & $3,36 \pm 0,72^{\mathrm{b}}$ & $3,32 \pm 0,69^{\mathrm{b}}$ & $3,94 \pm 1,01^{\mathrm{a}}$ \\
Distancia inserción PI $(\mu \mathrm{m})$ & $0,13 \pm 0,08^{\mathrm{b}}$ & $0,14 \pm 0,87^{\mathrm{b}}$ & $0,35 \pm 0,18^{\mathrm{a}}$ & $0,16 \pm 0,10^{\mathrm{b}}$ \\
Ángulo inserción PI $\left(^{\circ}\right)$ & $3,23 \pm 2,54^{\mathrm{b}}$ & $3,21 \pm 2,23^{\mathrm{b}}$ & $6,51 \pm 4,32^{\mathrm{a}}$ & $3,56 \pm 2,98^{\mathrm{b}}$ \\
\hline
\end{tabular}

DS: desviación estándar. SP: subpoblación. PI: pieza intermedia. ${ }^{a-d}$ Superíndices distintos dentro de fila indican diferencias significativas entre subpoblaciones espermáticas $(\mathrm{p}<0,05)$. / SD: standard deviation. SP: subpopulation. PI: mid piece ${ }^{\mathrm{a}-\mathrm{d}}$ Different superscripts within row indicate significant differences between sperm subpopulations $(\mathrm{p}<0.05)$. 

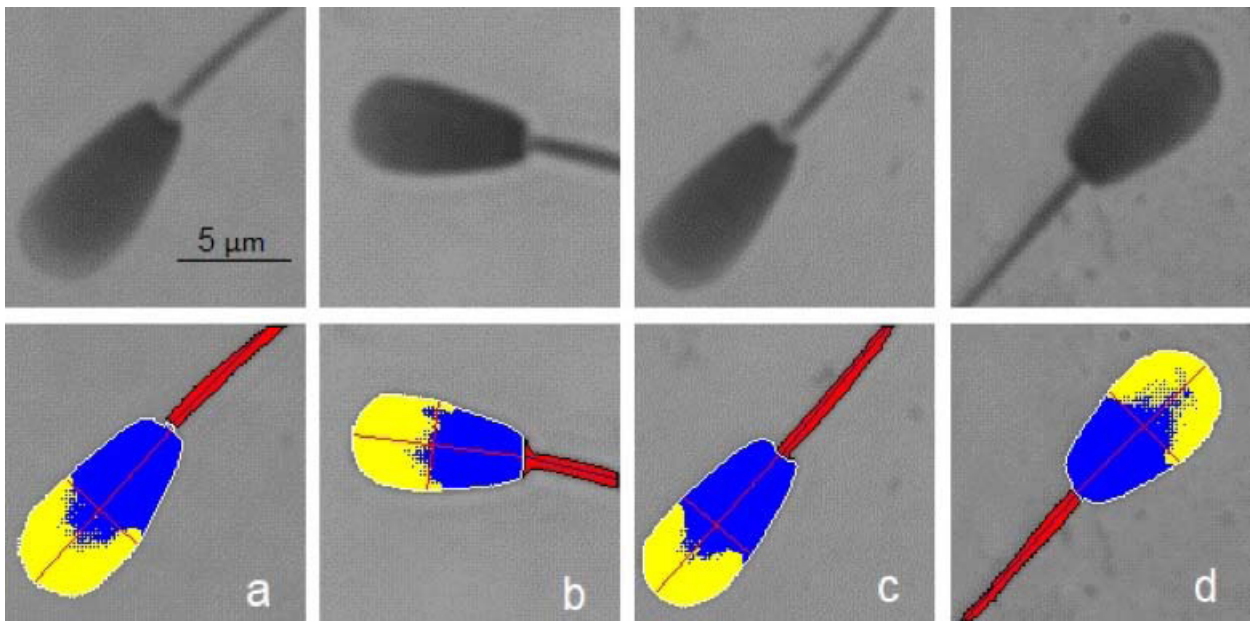

Figura 2. Espermatozoides de Odocoileus virginianus peruvianus que representan cada subpoblación. (a) SP1, grandes y alargadas; (b) SP2, células pequeñas y delgadas; (c) SP3, células alargadas, valores altos de elongación y elipticidad; (d) SP4, células de tamaño intermedio. Laboratorio de Biotecnologías Reproductivas, Granja K’ayra, Escuela Profesional de Zootecnia, Facultad de Ciencias Agrarias de la Universidad Nacional de San Antonio Abad del Cusco (UNSAAC), Cusco, Perú, 2016.

Fila superior, imágenes capturadas del microscopio. Fila inferior, imágenes digitalizadas: amarillo, acrosoma; azul, región post acrosomal (valores de la cabeza son la suma de ambas áreas); rojo, pieza intermedia. SP: subpoblación. Escala de barra $=5 \mu \mathrm{m}$, aplicable a todas las imágenes.

Figure 2. Odocoileus virginianus peruvianus spermatozoa representing each subpopulation. (a) SP1, large and elongated; (b) SP2, small and thin cells; (c) SP3, elongated cells, high elongation and ellipticity values; (d) SP4, cells of intermediate size. Reproductive Biotechnologies Laboratory, K'ayra Farm, Professional School of Animal Sciences, Faculty of Agrarian Sciences at National University of San Antonio Abad in Cuzco (UNSAAC), Cusco, Perú, 2016.

Top row, images captured from the microscope. Bottom row, digitized images: yellow, acrosome; blue, post acrosomal region (head values are the sum of both areas); red, intermediate piece. SP: subpopulation. Bar scale $=5 \mu \mathrm{m}$, it applies to all images.

\section{Discusión}

En este estudio se analizó la morfometría de 740 espermatozoides epididimarios de venado de cola blanca (Odocoileus virginianus peruvianus) y se determinaron diferencias significativas $(\mathrm{p}<0,05)$ entre machos para las variables de tamaño y forma de la cabeza como anchura, elipticidad, elongación y regularidad de la cabeza espermática. Además, se determinaron diferencias para variables de anchura, distancia y ángulo de inserción de la pieza intermedia. Resultados similares fueron reportados en otra subespecie del venado de cola blanca (HernándezCorredor et al., 2018), aunque la tinción utilizada en el estudio fue diferente, en venado de las pampas (Beracochea et al., 2014) y en ciervo rojo ibérico (Esteso et al., 2006; Soler et al., 2005). Estos últimos autores utilizaron tres tinciones, de las cuales una fue similar a la empleada en este trabajo (Diff Quik).

Las diferencias podrían estar relacionadas con factores ambientales y/o genéticos como la edad, y la dominancia (Esteso et al., 2006). Otros autores han propuesto que existen factores intrínsecos y extrínsecos que afectan los resultados de morfometría espermática. Dentro de los factores intrínsecos se incluyen los factores genéticos, edad y madurez sexual de los individuos, mientras que entre los factores extrínsecos está la influencia del medio ambiente, la nutrición, el manejo y el método de tinción e iluminación de la lámpara (Yániz, Soler et al., 2015).

En relación con las medidas morfométricas del espermatozoide de venado de cola blanca, los valores que se obtuvieron para la subespecie peruvianus, fueron inferiores en la longitud, anchura, área y perímetro de la cabeza, 
a las reportadas en otra subespecie del venado cola blanca (Hernández-Corredor et al., 2018). En ambos estudios se utilizó el mismo sistema CASA de análisis computarizado ISAS ${ }^{\circledast}$, pero los medios de tinción celular fueron diferentes, lo que podría haber influido en los resultados como ha sido asociado en otros trabajos realizados.

$\mathrm{Al}$ caracterizar las células espermáticas sobre el venado de las pampas (Ozotoceros bezoarticus), se han reportado valores morfométricos del área de la cabeza $\left(28,1 \mu \mathrm{m}^{2}\right)$, longitud $(7,6 \mu \mathrm{m})$ y perímetro $(21,9 \mu \mathrm{m}), \mathrm{y}$ valores similares de rugosidad $(0,7)$, elongación $(0,3)$ y regularidad $(0,9)$ (Beracochea et al., 2014); medidos con la misma tinción que en el presente trabajo, aunque un sistema CASA-Morph diferente. Al evaluar diferentes métodos de tinción del espermatozoide del ciervo rojo ibérico, se han reportado valores de longitud y perímetro de la cabeza del espermatozoide, similares a los que se reportan en este estudio, con el mismo sistema CASA-Morph (Soler et al., 2005). No se han reportado resultados de las medidas de la anchura, área, distancia y ángulo de inserción de la pieza intermedia del espermatozoide, de alguna subespecie del venado de cola blanca, ni tampoco de otros cérvidos como el venado de las pampas o el ciervo rojo ibérico, por lo que los datos encontrados no se pueden discutir.

Este es el primer estudio que ofrece información de la estructura de subpoblaciones espermáticas en función a la morfometría en venados de cola blanca de la subespecie peruvianus, al identificar cuatro subpoblaciones espermáticas definidas por criterios de tamaño y forma de la cabeza y el ángulo de inserción de la pieza intermedia del espermatozoide. En cérvidos como el venado de las pampas se han reportado tres subpoblaciones (SP) en función de su morfometría con procedimientos multivariados de análisis similares a los del presente estudio (Beracochea et al., 2014). En ciervo rojo ibérico, se reportaron 3 SP luego de realizar diferentes técnicas de análisis como vectores de soporte (SVM) (Ramón et al., 2013). Además, los espermatozoides más pequeños tienden a presentar una mejor resistencia durante el proceso de criopreservación (Esteso et al., 2006). En el presente estudio, las células con el patrón antes descrito correspondieron a la SP2, sin embargo, es necesario más investigación para determinar una relación directa entre los patrones morfométricos del espermatozoide del venado cola blanca y la resistencia a los procesos de criopreservación. La distribución desigual de espermatozoides en las diferentes subpoblaciones refuerza la idea de la heterogeneidad del eyaculado en su conformación. La mayor parte de los espermatozoides se agruparon en la SP2, donde las células presentaron cabezas pequeñas o alargadas. Esto refuerza la idea de una célula pequeña y elíptica con mayor capacidad de movilización como mecanismo evolutivo de capacidad fecundante.

La heterogeneidad en los porcentajes de espermatozoides en cada una de las subpoblaciones, también ha sido reportada por Beracochea et al. (2014) y Ramón et al. (2013), en el venado de las pampas y el ciervo rojo ibérico, respectivamente, y en otros mamíferos como el vacuno (Valverde et al., 2016), perro (Soler, Alambiaga et al., 2017) o llama (Cucho et al., 2021), esta heterogeneidad de espermatozoides garantizaría un mayor potencial para fecundar un ovocito a intervalos impredecibles después de la eyaculación (Curry, 2000), esta es una estrategia de los espermatozoides para lograr una fertilización exitosa.

\section{Conclusiones}

La caracterización de los espermatozoides epididimarios del venado cola blanca analizados con un sistema CASA-Morph permitió identificar cuatro subpoblaciones espermáticas con diferentes patrones morfométricos relacionados con el tamaño, forma de la cabeza e inserción de la pieza intermedia del espermatozoide.

Este trabajo es el primero en caracterizar la morfometría del espermatozoide del venado cola blanca y puede ser la base de futuros trabajos de investigación en programas de reproducción asistida de esta especie con propósitos y fines de conservación.

Agron. Mesoam. 33(2): Artículo 46938, 2022 ISSN 2215-3608 doi:10.15517/am.v33i2.46938 


\section{Agradecimientos}

Los autores agradecen al Laboratorio de Biotecnologías Reproductivas del Centro Experimental La Raya de la Universidad Nacional de San Antonio Abad del Cusco (UNSAAC), por las facilidades aportadas durante la presente investigación.

\section{Referencias}

Ake-Lopez, J., Cavazos-Arizpe, E., Magana-Monforte, J. G., Centurion-Castro, F., \& Silva-Mena, C. (2010). Effect of age and postmortem time on some White-Tailed deer (Odocoileus virginianus texanus) epididymal sperm characteristics and response of cryopreservation. American Journal of Animal and Veterinary Sciences, 5(3), 183-186. https://doi. org/10.3844/ajavsp.2010.183.186https://doi.org/10.3844/ajavsp.2010.183.186

Barquero, V., Roldan, E. R. S., Soler, C., Yániz, J. L., Camacho, M., \& Valverde, A. (2021). Predictive Capacity of Boar Sperm Morphometry and Morphometric Sub-Populations on Reproductive Success after Artificial Insemination. Animals, 11(4), Article 920. https://doi.org/10.3390/ani11040920

Barquero, V., Víquez, L., Calderón-Calderón, J. C., \& Valverde, A. (2021). Optimal frame rate to evaluate boar sperm kinematic with a CASA-Mot system. Agronomía Mesoamericana, 32(1), 1-18. https://doi.org/10.15517/am.v32i1.41928

Belleannée, C., Thimon, V., \& Sullivan, R. (2012). Region-specific gene expression in the epididymis. Cell and Tissue Research, 349, 717-731. https://doi.org/10.1007/s00441-012-1381-0

Beracochea, F., Gil, J., Sestelo, A., Garde, J. J., Santiago-Moreno, J., Fumagalli, F., \& Ungerfeld, R. (2014). Sperm characterization and identification of sperm sub-populations in ejaculates from pampas deer (Ozotoceros bezoarticus). Animal Reproduction Science, 149(3-4), 224-230. https://doi.org/10.1016/j.anireprosci.2014.07.013

Cooper, T. G., \& Yeung, C. -H. (2006). Sperm maturaiton in the human epididymis. In C. J. De Jonge \& C. Barratt (Eds.), The sperm cell production, maturation, fertilization, regeneration (pp. 72-107). Cambridge University Press. https://doi. org/10.1017/CBO9780511545115.005

Cornwall, G. A. (2009). New insights into epididymal biology and function. Human Reproduction Update, 15(2), $213-227$. https://doi.org/10.1093/humupd/dmn055

Cucho, H., Gallegos, M., Ccoiso, R., Meza, A., Ampuero, E., Ordóñez, C., \& Valverde, A. (2021). Morphometry and subpopulation of llama (Lama glama) sperm using the ISAS® CASA-Morph system. Revista de Investigaciones Veterinarias Del Peru, 32(1), Article e19506. https://doi.org/10.15381/RIVEP.V32I1.19506

Cucho, H., López, Y., Caldeira, C., Valverde, A., Ordóñez, C., \& Soler, C. (2019). Comparison of three different staining methods for the morphometric characterization of Alpaca (Vicugna pacos) sperm , using ISAS ® CASA-Morph system. Nova Biologica Reperta, 6(3), 284-291. https://doi.org/10.29252/nbr.6.3.284

Curry, M. R. (2000). Cryopreservation of semen from domestic livestock. Reviews of Reproduction, 5(1), 46-52. https://doi. org/10.1530/ror.0.0050046

Downing-Meisner, A., Klaus, A. V., \& O’Leary, M. A. (2005). Sperm head morphology in 36 species of artiodactylans, perissodactylans, and cetaceans (Mammalia). Journal of Morphology, 263(2), 179-202. https://doi.org/10.1002/ jmor.10297

Agron. Mesoam. 33(2): Artículo 46938, 2022

ISSN 2215-3608 doi:10.15517/am.v33i2.46938 
Esteso, M. C., Fernández-Santos, M. R., Soler, A. J., Montoro, V., Martínez-Pastor, F., \& Garde, J. J. (2009). Identification of sperm-head morphometric subpopulations in iberian red deer epididymal sperm samples. Reproduction in Domestic Animals, 44(2), 206-211. https://doi.org/10.1111/j.1439-0531.2007.01029.x

Esteso, M. C., Rodríguez, E., Toledano-Díaz, A., Castaño, C., Pradiee, J., López-Sebastián, A., \& Santiago-Moreno, J. (2015). Descriptive analysis of sperm head morphometry in Iberian ibex (Capra pyrenaica): Optimum sampling procedure and staining methods using Sperm-Class Analyzer ${ }^{\circledR}$. Animal Reproduction Science, 155(1), 42-49. https://doi. $\operatorname{org} / 10.1016 /$ j.anireprosci.2015.01.014

Esteso, M. C., Soler, A. J., Fernández-Santos, M. R., Quintero-Moreno, A. A., \& Garde, J. J. (2006). Functional significance of the sperm head morphometric size and shape for determining freezability in Iberian red deer (Cervus elaphus hispanicus) epididymal sperm samples. Journal of Andrology, 27(5), 662-670. https://doi.org/10.2164/jandrol.106.000489

Esteso, M. C., Toledano-Díaz, A., Castaño, C., Pradiee, J., Lopez-Sebastián, A., \& Santiago-Moreno, J. (2018). Effect of two cooling protocols on the post-thaw characteristics of Iberian ibex sperms. Cryobiology, 80(1), 12-17. https://doi. org/10.1016/j.cryobiol.2018.01.003

Gaddum, P. (1968). Sperm maturation in the male reproductive tract: Development of motility. The Anatomical Record, 161(4), 471-482. https://doi.org/10.1002/ar.1091610409

Gage, M. (1998). Mammalian sperm morphometry. Proceedings of the Royal Society B: Biological Sciences, 265(1391), 97-103. https://doi.org/10.1098/rspb.1998.0269

Hernández-Corredor, L., Silva-Torres, A., Celis-Alba, D., Landaeta-Hernández, A., \& Rubio-Parada, J. (2018). Evaluación de la motilidad y morfometría en espermatozoides descongelados de venado cola blanca (Odocoileus virginianus). ResearchGate. https://n9.cl/w5eb8

Hidalgo, M., Rodríguez, I., Dorado, J., \& Soler, C. (2008). Morphometric classification of Spanish thoroughbred stallion sperm heads. Animal Reproduction Science, 103(3-4), 374-378. https://doi.org/10.1016/j.anireprosci.2007.06.001

Kaiser, H. F. (1958). The varimax criterion for analytic rotation in factor analysis. Psychometrika, 23(3), 187-200. https://doi. $\operatorname{org} / 10.1007 / \mathrm{BF} 02289233$

Kaufman, L., \& Rousseeuw, P. J. (2005). Finding groups in data : an introduction to cluster analysis. John Wiley Sons, Inc., Publications. https://doi.org/10.1002/9780470316801

Kirschner, S. M., \& Rodenkirch, R. (2017). Assessment of butorphanol-azaperone-medetomidine combination as anesthesia for semen collection and evaluation of semen quality in white-tailed deer (Odocoileus virginianus). Animal Reproduction Science, 184(1), 196-203. https://doi.org/10.1016/j.anireprosci.2017.07.016

Maroto-Morales, A., García-Álvarez, O., Ramón, M., Martínez-Pastor, F., Fernández-Santos, M. R., Soler, A., \& Garde, J. J. (2016). Current status and potential of morphometric sperm analysis. Asian Journal of Andrology, 18(6), 863-870. https://doi.org/10.4103/1008-682X.187581

Martí, J. I., Aparicio, I. M., Leal, C. L. V., \& García-Herreros, M. (2012). Seasonal dynamics of sperm morphometric subpopulations and its association with sperm quality parameters in ram ejaculates. Theriogenology, 78(3), 528-541. https://doi.org/10.1016/j.theriogenology.2012.02.035

Martinez-Pastor, F., Diaz-Corujo, A. R., Anel, E., Herraez, P., Anel, L., \& de Paz, P. (2005). Post mortem time and season alter 
subpopulation characteristics of Iberian red deer epididymal sperm. Theriogenology, 64(4), 958-974. https://doi. org/10.1016/j.theriogenology.2005.01.003

Martinez-Pastor, F., Garcia-Macias, V., Alvarez, M., Chamorro, C., Herraez, P., de Paz, P., \& Anel, L. (2006). Comparison of two methods for obtaining spermatozoa from the cauda epididymis of Iberian red deer. Theriogenology, 65(3), 471-485. https://doi.org/10.1016/j.theriogenology.2005.05.045

Martinez-Pastor, F., Garcia-Macias, V., Alvarez, M., Herraez, P., Anel, L., \& de Paz, P. (2005). Sperm Subpopulations in Iberian Red Deer Epididymal Sperm and Their Changes Through the Cryopreservation Process. Biology of Reproduction, 72(2), 316-327. https://doi.org/10.1095/biolreprod.104.032730

Ministerio de Desarrollo Agrario y Riego del Perú. (2014). Decreto Supremo que aprueba la actualización de la lista de clasificación y categorización de las especies amenazadas de fauna silvestre legalmente protegidas $\left(\mathrm{N}^{\circ}\right.$ 004-2014-MINAGRI). Organización de las Naciones Unidas para la Alimentación y la Agricultura. http:// extwprlegs1.fao.org/docs/pdf/per132692.pdf

Murtagh, F., \& Legendre, P. (2014). Ward's Hierarchical Agglomerative Clustering Method: Which Algorithms Implement Ward's Criterion? Journal of Classification, 31(3), 274-295. https://doi.org/10.1007/s00357-014-9161-z

Orgebin-Crist, M. C. (1967). Sperm maturation in rabbit epididymis. Nature, 216(5117), 816-818. https://doi. org/10.1038/216816a0

Pacheco, V., Cadenillas, R., Salas, E., Tello, C., \& Zeballos, H. (2009). Diversidad y endemismo de los mamíferos del Perú. Revista Peruana de Biología, 16(1), 5-32. https://doi.org/10.15381/rpb.v16i1.111

Peña, F., Saravia, F., García-Herreros, M., Núñez-Martínez, I., Tapia, J., Johannisson, A., Wallgren, M., \& Rodríguez-Martínez, H. (2005). Identification of sperm morphometric subpopulations in two different portions of the boar ejaculate and its relation to postthaw quality. Journal of Andrology, 26(6), 716-723. https://doi.org/10.2164/jandrol.05030

Peña Hernández, A. E. (2014). Evaluación objetiva de la motilidad de los espermatozoides epididimarios de ciervo ibérico. Relaciones con la congelabilidad y la calidad del semen [Tesis de Doctorado, Universidad de CastillaLa Mancha]. Repositorio institucional del Consejo Superior de Investigaciones Científicas https:/digital.csic.es/ handle/10261/147311

Ramírez Lozano, R. G. (2012). Alimentación Del Venado Cola Blanca: Biología y Ecología Nutricional. Palibrio.

Ramón, M., Martínez-Pastor, F., García-Álvarez, O., Maroto-Morales, A., Soler, A. J., Jiménez-Rabadán, P., FernándezSantos, M. R., Bernabéu, R., \& Garde, J. J. (2012). Taking advantage of the use of supervised learning methods for characterization of sperm population structure related with freezability in the Iberian red deer. Theriogenology, 77(8), 1661-1672. https://doi.org/10.1016/j.theriogenology.2011.12.011

Ramón, M., Soler, A. J., Ortiz, J. A., García-Alvarez, O., Maroto-Morales, A., Roldan, E. R. S., \& Garde, J. J. (2013). Sperm population structure and male fertility: An intraspecific study of sperm design and velocity in Red Deer. Biology of Reproduction, 89(5), 1-7. https://doi.org/10.1095/biolreprod.113.112110

Robaire, B., Hinton, B., \& Orgebin-Crist, M. (2015). Knobil and Neill's Physiology of Reproduction. In T. M. Plant \& A. J. Zeleznik (Eds.), Physiology of reproduction (4 ${ }^{\text {th }}$ Ed., pp. 691-771). Elsevier Academic Press. https://doi.org/10.1016/ B978-0-12-397175-3.15001-X

Agron. Mesoam. 33(2): Artículo 46938, 2022

ISSN 2215-3608 doi:10.15517/am.v33i2.46938 
Soler, C., Alambiaga, A., Martí, M. A., García-Molina, A., Valverde, A., Contell, J., \& Campos, M. (2017). Dog sperm head morphometry: its diversity and evolution. Asian Journal of Andrology, 19(2), 149-153. https://doi.org/10.4103/1008682X.189207

Soler, C., Contell, J., Bori, L., Sancho, M., García-Molina, A., Valverde, A., \& Segarvall, J. (2017). Sperm kinematic, head morphometric and kinetic-morphometric subpopulations in the blue fox (Alopex lagopus). Asian Journal of Andrology, 19(2), 154-159. https://doi.org/10.4103/1008-682X.188445

Soler, C., Cooper, T., Valverde, A., \& Yániz, J. (2016). Afterword to Sperm morphometrics today and tomorrow special issue in Asian Journal of Andrology. Asian Journal of Andrology, 18(6), 895-897. https://doi.org/10.4103/1008-682X.188451

Soler, C., Gadea, B., Soler, A., Fernández-Santos, M., Esteso, M., Núñez, J., Moreira, P., Núñez, M., Gutiérrez, R., Sancho, M., \& Garde, J. (2005). Comparison of three different staining methods for the assessment of epididymal red deer sperm morphometry by computerized analysis with ISAS®. Theriogenology, 64(5), 1236-1243. https://doi.org/10.1016/j. theriogenology.2005.02.018

Souza, T. (2009). Avaliação andrológica e criopreservação de sêmen de pumas (Puma concolor Linnaeus, 1771) adultos [Magister Dissertation, Universidad Federal de Viçosa] Repositório do Universidad Federal de Viçosa. https://www. locus.ufv.br/bitstream/123456789/4980/1/texto\%20completo.pdf

Spencer, N. H. (2013). Essentials of multivariate data analysis. Chapman and Hall/CRC Press. https://doi.org/10.1201/b16344

Thurston, L., Watson, P., Mileham, A., \& Holt, W. (2001). Morphologically distinct sperm subpopulations defined by Fourier shape descriptors in fresh ejaculates correlate with variation in boar semen quality following cryopreservation. Journal of Andrology, 22(3), 382-394. http://www.ncbi.nlm.nih.gov/pubmed/11330638

Tuset, V. M., Dietrich, G. J., Wojtczak, M., Słowińska, M., de Monserrat, J., \& Ciereszko, A. (2008). Comparison of three staining techniques for the morphometric study of rainbow trout (Oncorhynchus mykiss) spermatozoa. Theriogenology, 69(8), 1033-1038. https://doi.org/10.1016/j.theriogenology.2008.01.012

Valverde, A., Arenán, H., Sancho, M., Contell, J., Yániz, J., Fernández, A., \& Soler, C. (2016). Morphometry and subpopulation structure of Holstein bull spermatozoa: variations in ejaculates and cryopreservation straws. Asian Journal of Andrology, 18(6), 851-857. https://doi.org/10.4103/1008-682X.187579

Valverde, A., Barquero, V., \& Soler, C. (2020). The application of computer-assisted semen analysis (CASA) technology to optimise semen evaluation. A review. Journal of Animal and Feed Sciences, 29(3), 189-198. https://doi.org/10.22358/ jafs/127691/2020

Valverde, A., Castro-Morales, O., Madrigal-Valverde, M., Camacho, M., Barquero, V., Soler, C., \& Roldan, E. R. S. (2021). Sperm kinematic subpopulations of the American crocodile (Crocodylus acutus). PLOS ONE, 16(3), Article e0248270. https://doi.org/10.1371/journal.pone.0248270

Valverde, A., Castro-Morales, O., Madrigal-Valverde, M., \& Soler, C. (2019). Sperm kinematics and morphometric subpopulations analysis with CASA systems: A review. Revista de Biología Tropical, 67(6), 1473-1487. https://doi. org/10.15517/rbt.v67i6.35151

Valverde, A., Madrigal-Valverde, M., Camacho-Calvo, M., Zambrana-Jiménez, A., \& López, L. (2018). Efecto de la composición racial sobre la calidad espermática de verracos. Agronomía Mesoamericana, 29(3), 485-506. https://doi. org/10.15517/ma.v29i3.32445

Agron. Mesoam. 33(2): Artículo 46938, 2022 ISSN 2215-3608 doi:10.15517/am.v33i2.46938 
Valverde, A., Madrigal-Valverde, M., Castro-Morales, O., Gadea-Rivas, A., Johnston, S., \& Soler, C. (2019). Kinematic and head morphometric characterisation of spermatozoa from the Brown Caiman (Caiman crocodilus fuscus). Animal Reproduction Science, 207(1), 9-20. https://doi.org/10.1016/J.ANIREPROSCI.2019.06.011

Víquez, L., Barquero, V., Soler, C., Roldan, E. R. S., \& Valverde, A. (2020). Kinematic Sub-Populations in Bull Spermatozoa: A Comparison of Classical and Bayesian Approaches. Biology, 9(6), 138-154. https://doi.org/10.3390/biology9060138

Yániz, J. L., Palacín, I., Vicente-Fiel, S., Sánchez-Nadal, J. A., \& Santolaria, P. (2015). Sperm population structure in high and low field fertility rams. Animal Reproduction Science, 156, 128-134. https://doi.org/10.1016/j.anireprosci.2015.03.012

Yániz, J. L., Soler, C., \& Santolaria, P. (2015). Computer assisted sperm morphometry in mammals: A review. Animal Reproduction Science, 156, 1-12. https://doi.org/10.1016/J.ANIREPROSCI.2015.03.002

Yániz, J. L., Vicente-Fiel, S., Soler, C., Recreo, P., Carretero, T., Bono, A., Berné, J. M., \& Santolaria, P. (2016). Comparison of different statistical approaches to evaluate morphometric sperm subpopulations in men. Asian Journal of Andrology, 18(6), 819-823. https://doi.org/10.4103/1008-682X.186872 